\title{
Pressure ulcer healing with an intensive nutrition intervention in an acute setting: a pilot randomised controlled trial
}

Objective: To investigate the feasibility of recruitment, retention, intervention delivery and outcome measurement in a nutritional intervention to promote pressure ulcer healing in an acute setting. Method: Some 50 tertiary hospital patients with stage II or greater pressure ulcer were randomised to receive either individualised nutritional care by a dietitian, including prescription of wound healing supplements; or standard nutritional care. Relevant nutritional and pressure ulcer (PU) parameters were collected at day 5, 10, 15, 22 and then weekly or until discharge.

Results: The median length of hospital stay was 14 days (1-70) with 29 patients discharged by day 15 . There were 24 patients discharged before their PU fully healed. Per cent change in valid PU area and score measures from baseline to day 15 were chosen for outcome data analysis to account for varying initial size and severity

\begin{abstract}
of the wound and length of stay. There was a larger percentage reduction in PU measures in the intervention group, but this was not statistically significant. Little difference was found in nutritional intake between the control and intervention groups indicating a requirement to focus on effective delivery of the intervention in future studies. Future studies in the acute setting need to account for length of stay and ideally follow patients until full healing.

Conclusion: Results indicate a positive association with nutrition intervention and PU healing and that a rigorously designed and adequately powered study is feasible.

Declaration of interest: This research was supported by a grant from the Queensland Health, Health Practitioner Research Scheme. The authors have no conflicts of interest to declare.
\end{abstract}

Pressure ulcer $\bullet$ nutrition intervention $\bullet$ acute care

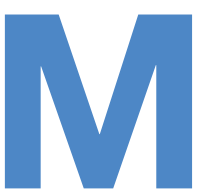

alnutrition has been found to be associated with a significantly higher risk of developing a pressure ulcer (PU). ${ }^{1-3}$ Targeted nutritional interventions, such as provision of nutritional supplementation, have been found to reduce the incidence of $\mathrm{PU}$ in at-risk patients, ${ }^{4}$ and this was shown to be a cost-effective approach to the prevention of PU. 5

Few high-quality studies have investigated nutritional interventions in the treatment of established PU. Systematic reviews of nutrition support in the treatment of PU describe a nonsignificant trend towards enhanced healing, especially with a high protein formula or formulae marketed as 'wound healing' (enriched with arginine, vitamin C and zinc). ${ }^{4,6}$ However, sample sizes of the studies were small and lacked statistical power, or were of poor methodological quality. The most recent Cochrane

M.D. Banks, ${ }^{1}$ PhD AdvAPD; L.J. Ross, ${ }^{1}$ PhD AdvAPD; J. Webster, ${ }^{1}$ BA RN; A. Mudge,${ }^{2}$ PhD MBBS; M. Stankiewicz, ${ }^{3}$ RN; K. Dwyer, ${ }^{1}$ B Health Sc (Nutr \& Diet) APD; K. Coleman, ${ }^{4}$ M Nursing; J. Campbell, ${ }^{4} \mathrm{RN}$

E-mail: merrilyn.banks@health.qld.gov.au

1 Department of Nutrition \& Dietetics, Royal Brisbane \& Women's Hospital, Herston, QLD.

2 Internal Medicine \& Aged Care, Royal Brisbane \& Women's Hospital, Herston, QLD.

3 Centre for Research \& Education in Nursing, Royal Brisbane \& Women's Hospital, Herston, QLD. 4 Skin Integrity Services Royal Brisbane \& Women's Hospital, Herston, QLD. systemic review ${ }^{6}$ concluded that there is no clear evidence of a benefit with nutrition interventions in the treatment of PUs, and that further trials of high methodological quality are necessary. Recently a multicentre, randomised, controlled, blinded trial was conducted in 200 malnourished patients from longterm care and home care services. This found supplementation with 'wound healing formula' resulted in a greater reduction in PU area compared with control formula at eight weeks. ${ }^{7}$ Observational studies of nutritional requirements and intake of patients with PU and chronic wounds indicate that protein, energy and micronutrient intake often do not meet requirements and many patients are malnourished as a consequence. ${ }^{8-10}$ It therefore remains unclear whether nutritional intervention in the acute hospital setting is effective in enhancing the healing of PU. Furthermore, it is still unknown if patients' nutritional needs remain unmet due to poor intake, and/or do patients with PU have increased nutritional requirements, if so, what type of intervention is most likely to be effective and/or do pharmacological intakes of particular nutrients enhance healing? Answers to these clinical questions are urgently needed, so that effective dietary approaches to PU treatment become an established part of routine care. However, there are significant challenges to designing trials of nutrition intervention 
to promote $\mathrm{PU}$ healing. PU are most prevalent in vulnerable older patients who may be difficult to recruit to clinical trials and comorbidity may introduce important confounders. In an acute environment, patients may be discharged before PU healing, making measuring outcomes difficult. Efficacy of nutrition intervention will depend on the effective delivery of the intervention as part of complex care regimens.

The aim of the study was to investigate the feasibility of recruitment, retention, intervention delivery and outcome measurement in a nutritional intervention intended to promote PU healing in an acute care setting, to inform a future, adequately powered, randomised controlled trial (RCT).

\section{Methods}

Participants were inpatients of a tertiary referral hospital in Brisbane, Australia. Eligible patients with a stage II-IV PU, either existing on admission or acquired during admission, were identified to the research nurse for potential recruitment by wardbased clinicians. Patients excluded were those:

- Unable to receive nutrition support via the enteral route (on parenteral nutrition)

- Inappropriate for intensive nutrition support (patients receiving palliative care or medically deteriorating)

- Unable to follow nutrition support advice (cognitively impaired, language barriers)
- Previously enrolled in the study.

The study conforms to the guidelines set out in the Declaration of Helsinki and was approved by the hospital Human Research Ethics Committee.

Written informed consent was obtained from eligible patients or their carers. After recruitment, a computer-generated randomised list was used to determine the group allocation sequence and group allocation was performed by telephoning an independent researcher. Randomisation was stratified by PU stage (stage 11 or stage III and IV) to ensure equal representation of PU severity across groups. Where more than one PU existed, the highest stage PU was chosen as the primary PU for data collection purposes.

Participants were randomised to either of the following:

- Standard nutritional care-provided by the clinical team which usually included a dietitian; and may have included a standard hospital diet or high protein/energy diet and/or nutritional supplements and/or enteral tube feeding

- Intensive individualised nutritional care-provided by a research dietitian; and included a high protein/ energy diet and/or supplements aimed at meeting estimated nutritional requirements of $1.2 \mathrm{~g}$ protein/ $\mathrm{kg}$ body weight/day and $30 \mathrm{kcal} / \mathrm{kg}$ per day and the prescription of a 'wound healing' nutritional formulae, enriched with arginine, vitamin C and zinc.

Fig 1. CONSORT flow chart

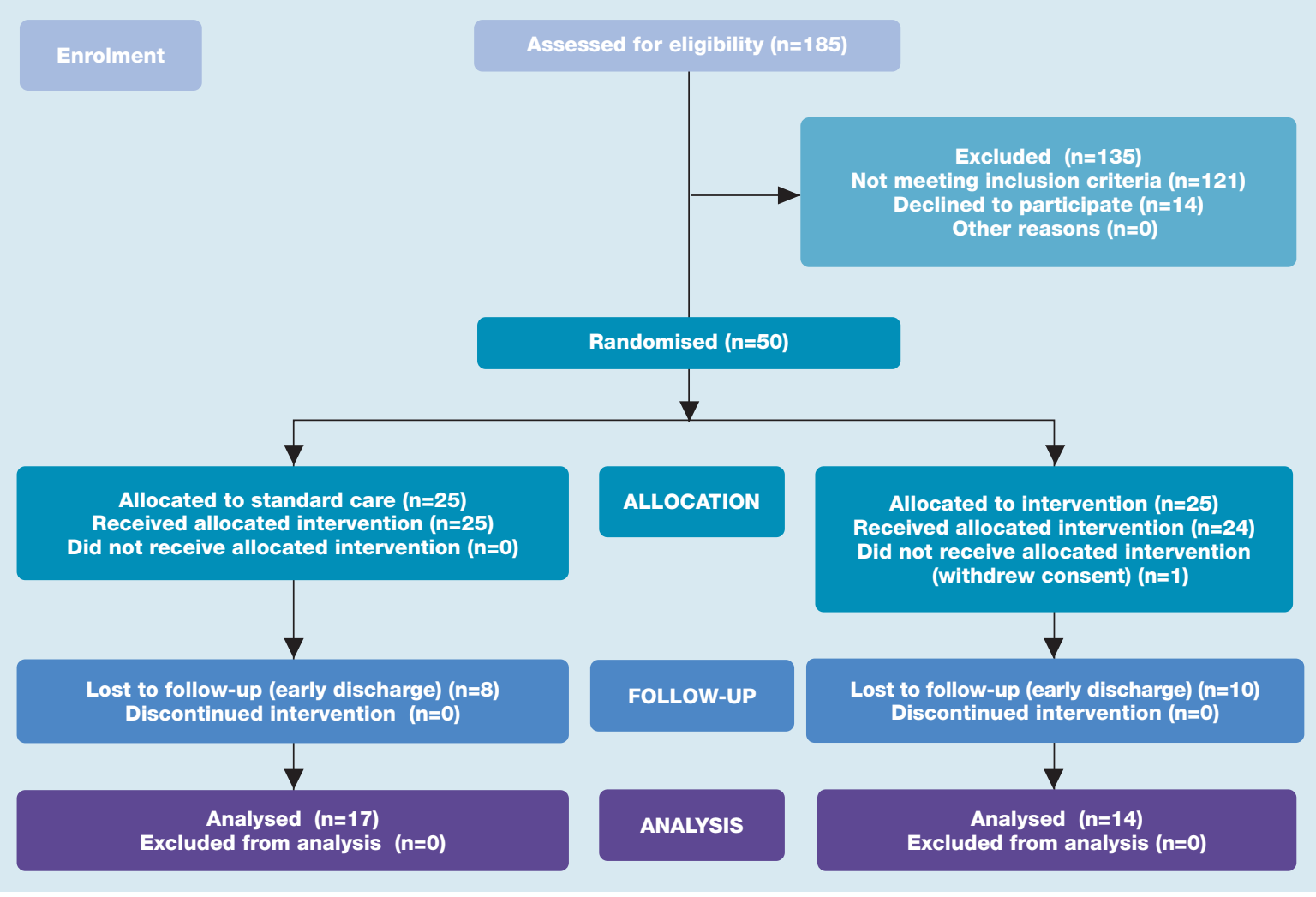


Table 1. Baseline characteristics for standard care and intervention groups

\begin{tabular}{|c|c|c|c|c|c|}
\hline \multirow[t]{2}{*}{ Parameter } & $\begin{array}{l}\text { Standard care } \\
n=25\end{array}$ & $\begin{array}{l}\text { Intervention } \\
n=25\end{array}$ & Parameter & $\begin{array}{l}\text { Standard care } \\
n=25\end{array}$ & $\begin{array}{l}\text { Intervention } \\
n=25\end{array}$ \\
\hline & \multicolumn{2}{|c|}{ Mean (SD) } & \multicolumn{3}{|l|}{ Number of PU present: } \\
\hline Age (years) & $65.8(15.8)$ & $62.3(20.7)$ & 1 & $14(56)$ & $16(64)$ \\
\hline Weight (kg) & 70.7 (15.3) & $79.2(23.3)$ & 2 & $8(32)$ & $7(28)$ \\
\hline \multirow[t]{2}{*}{ BMI $\left(\mathrm{kg} / \mathrm{m}^{2}\right)$} & $23.5(4.4)$ & $27.1(8.4)$ & $>3$ & $3(12)$ & $2(8)$ \\
\hline & \multicolumn{2}{|c|}{ n (\%) } & \multicolumn{3}{|c|}{ n (\%) } \\
\hline \multicolumn{3}{|l|}{ Gender: } & \multicolumn{3}{|l|}{ Stage of primary PU: } \\
\hline Female & $6(24)$ & $11(44)$ & ॥ & $11(44)$ & $12(48)$ \\
\hline Male & $19(76)$ & $14(56)$ & III & $7(28)$ & $8(32)$ \\
\hline \multirow{2}{*}{$\begin{array}{l}\text { BMI }\left(\mathbf{k g} / \mathbf{m}^{2}\right): \\
<20\end{array}$} & $n=24$ & $n=24$ & IV & $7(28)$ & $5(20)$ \\
\hline & $5(21)$ & $5(21)$ & \multicolumn{3}{|l|}{ Age of primary PU (days): } \\
\hline $20.1-25$ & $9(38)$ & $5(21)$ & 0-7 days & $11(44)$ & $12(48)$ \\
\hline $25.1-30$ & $9(38)$ & $8(33)$ & 8-14 days & $8(32)$ & $6(24)$ \\
\hline$>30$ & $1(4)$ & $6(25)$ & $15-30$ days & $3(12)$ & $4(16)$ \\
\hline \multirow{2}{*}{$\begin{array}{l}\text { Comorbidities: } \\
\text { Assoc with poor healing }\end{array}$} & $n=25$ & $n=25$ & $>30$ days & $3(12)$ & $3(12)$ \\
\hline & $6(24)$ & $9(36)$ & \multicolumn{3}{|l|}{ PU treatment: } \\
\hline Other & $11(44)$ & $7(28)$ & Moisture donating & $1(4)$ & $3(12)$ \\
\hline Nil & $8(32)$ & $9(36)$ & Absorb moisture & $21(84)$ & $14(56)$ \\
\hline \multicolumn{3}{|c|}{ Medications impeding healing: } & Antimicrobial & $1(4)$ & $6(24)$ \\
\hline Nil & $2(8)$ & $4(16)$ & Wound open - no dressing & $2(8)$ & $2(8)$ \\
\hline 1 & $10(40)$ & $10(40)$ & \multicolumn{3}{|l|}{ PUSH score: } \\
\hline$>1$ & $13(52)$ & $11(44)$ & $0-5$ & $3(12)$ & $4(16)$ \\
\hline \multicolumn{3}{|l|}{ Mobility: } & $6-11$ & $17(68)$ & $16(64)$ \\
\hline Cannot reposition & $12(48)$ & $5(20)$ & $12-17$ & $5(20)$ & $5(20)$ \\
\hline Can reposition but can't walk & $10(40)$ & $13(52)$ & \multicolumn{3}{|l|}{ PU area $\left(\mathrm{cm}^{2}\right)^{\star}:$} \\
\hline Can reposition and can walk & $3(12)$ & $7(28)$ & $0-5$ & $20(80)$ & $18(72)$ \\
\hline \multicolumn{3}{|l|}{ Nutritional status (SGA): } & $5.01-10$ & $0(0)$ & $5(20)$ \\
\hline Well nourished & $4(16)$ & $5(20)$ & $10.01-15$ & $0(0)$ & $2(8)$ \\
\hline Mild/moderate malnutrition & $15(60)$ & $13(52)$ & $15.01-20$ & $3(12)$ & $0(0)$ \\
\hline Severe malnutrition & $6(24)$ & $7(28)$ & $>=20.01$ & $2(8)$ & $0(0)$ \\
\hline \multicolumn{3}{|l|}{ Dietitian reviews / week } & \multicolumn{3}{|c|}{ Median (range) IQR } \\
\hline 0 & $13(52)$ & $12(48)$ & \multirow[t]{2}{*}{ PUSH score } & $7(4-17)$ & $9(5-14)$ \\
\hline 1 & $6(24)$ & $7(28)$ & & IQR: 6-11 & IQR: 6.5-9.0 \\
\hline $2-3$ & $6(24)$ & $6(24)$ & \multirow[t]{5}{*}{ PU area $\left(\mathrm{cm}^{2}\right)$} & $1.5(0.2-65.3)$ & $2.9(1-2.9)$ \\
\hline \multicolumn{3}{|l|}{ Diet prescription:* } & & IQR: 0.7-3.9 & IQR: $1-5.4$ \\
\hline High protein/energy & $17(68)$ & $10(40)$ & & & \\
\hline Standard & $2(8)$ & $9(36)$ & & & \\
\hline Restrictive (eg. diabetic) & $6(24)$ & $6(24)$ & & & \\
\hline
\end{tabular}

Participants were offered the choice of two different brands of the supplement based on their preference and prescription based on recommended daily dosage by manufacturer. It was expected that patients in the intensive care group would be reviewed by the research dietitian at least three times per week. All patients received evidence-based PU care according to local hospital practices.

The primary outcome was PU change, from baseline, in score and area, determined using the Pressure Ulcer Scale for Healing (PUSH) tool and VISITRAK (Smith \& Nephew), respectively. The PUSH tool was developed by the NPUAP as a quick reliable tool to monitor change in PU status over time. ${ }^{10}$ The tool provides a score from $0-17$ related to severity of the PU $(0=$ none) with sub-scores for area of PU (0-10), exudate amount (0-3; none-heavy) and tissue type (0-4; closednecrotic tissue). VISITRAK is a wound measurement 
Fig 2. Changes in $P U$ area $\left(\mathrm{cm}^{2}\right)$ across admission $P U$ area measures for individuals across admission for the control group (a) and the intervention group (b). Each line represents an individual patient
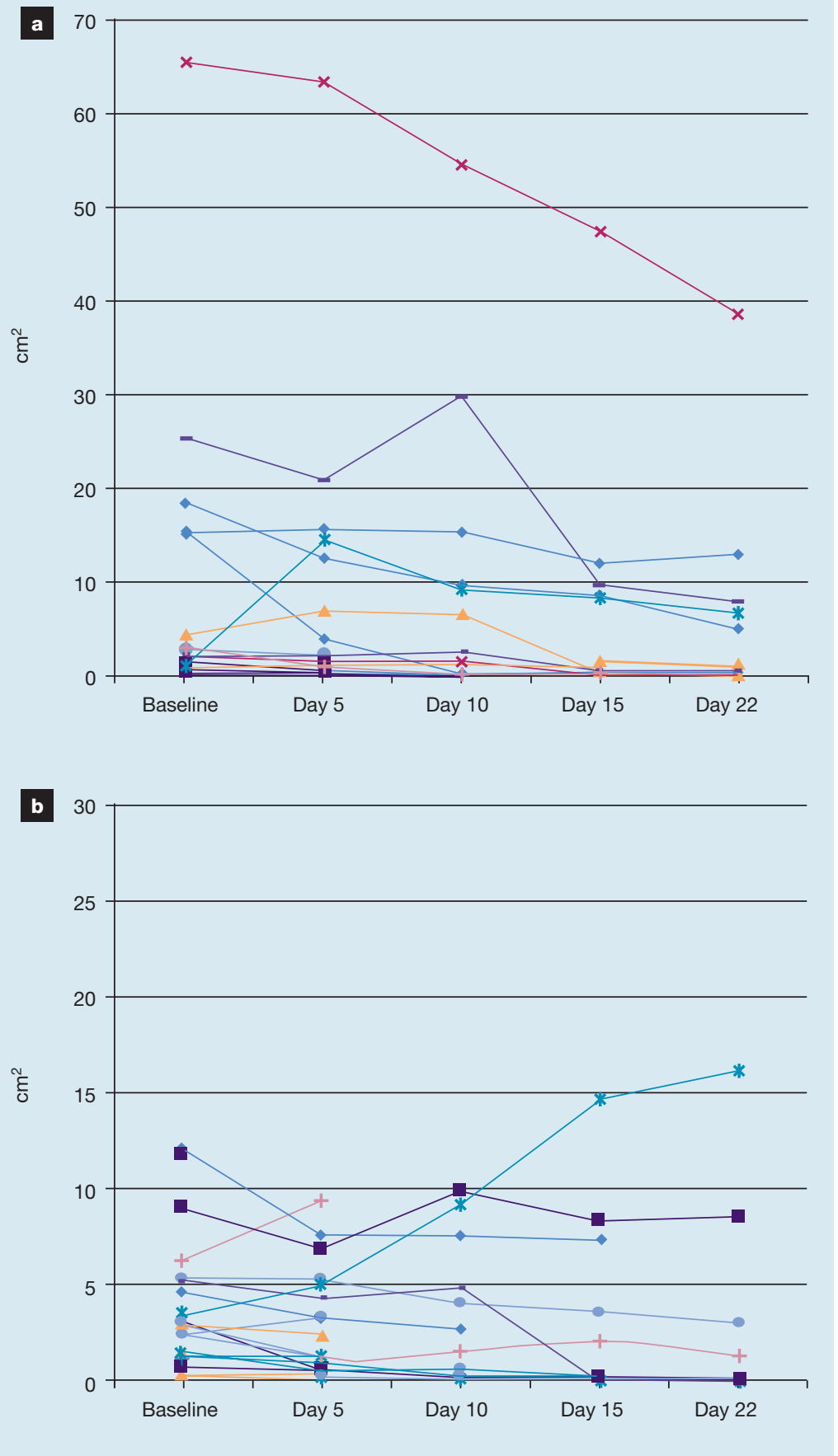

system which calculates wound area based on manual tracings of the wound. ${ }^{11}$ Other PU data collected included: number, stage, duration and location.

Demographic data collected for each patient included: age, gender, primary diagnostic group, comorbidities, smoking status, medications, mobility status and PU treatment methods. Nutrition-related data collected included: weight, body mass index
(BMI), nutritional status (using subjective global assessment), ${ }^{12}$ diet (standard, high protein/energy, restricted e.g. low fat), dietetic intervention (number of reviews per week), intake of protein and energy and supplements (from 24 hour food charts). Food charts were kept at the bedside to be completed by the patient or ward nurses, but were checked and completed if necessary by the research nurse with patient recall. Estimated nutritional requirements were determined as $1.5 \mathrm{~g}$ protein $/ \mathrm{kg} / \mathrm{day}$ and $30 \mathrm{kcal} /$ $\mathrm{kg} /$ day selected from within the range recommended by the National Pressure Ulcer Advisory Panel (NPUAP), European Pressure Ulcer Advisory Panel (EPUAP) and Pan Pacific Pressure Injury Alliance (PPPIA) Guidelines. ${ }^{13}$ The research nurse collected data collection on day $0,5,10,15,22$ and 29 ( \pm 1 day either side of the time point), and thereafter weekly, or until discharge from hospital. A range of pathological data were also collected where available. Length of hospital stay and healing status at discharge were also collected.

\section{Statistics}

Data were entered into SPSS for Windows 26 (Version 20.0, 2011, IBM Corp., Chicago IL. USA) for statistical analysis and reported as mean (SD) or median (minimum-maximum and interquartile range (IQR)) as applicable, and frequency (\%). Characteristics at baseline and during hospital stay were compared between groups, using $\chi$-square or independent t-tests, as appropriate. Correlation coefficients were used to assess the relationship between the different PU measurements. Changes in PU measurements were assessed using non-parametric tests for median data. Factors contributing to PU change were individually assessed using independent t-tests or one-way ANOVA when more than two categories were used. Significance was accepted at $\mathrm{p}<0.05$.

\section{Results}

Over an 8-month period, 185 patients were identified with a stage II-IV PU. There were 53 ineligible due to cognitive impairment, 30 for predicted early discharge or transfer, 28 for palliative care or medical deterioration, six on parenteral nutrition and four had been previously recruited. Furthemore, 14 eligible patients declined to participate, leaving 50 participants to be randomised. After baseline data was collected one patient withdrew. See Fig 1 Consort Diagram.

Characteristics of participants on recruitment are shown in Table 1. More than half of those recruited were admitted to hospital for surgical procedures $(n=28)$, others were for medical $(n=15)$ and oncology treatment $(n=7)$, with an even distribution across the randomised groups. Only 7 (14\%) participants were smokers, evenly distributed across the groups. There were more males $(n=33)$ recruited than females $(\mathrm{n}=17)$, with ages ranging from 23 to 95 years. On average, patients allocated to the intervention group 
were heavier, with more patients with a BMI greater than $30 \mathrm{~kg} / \mathrm{m}^{2}$ than those allocated to the standard care group. Factors that might impact on healing, such as comorbidities, medication use, immobility and malnutrition, were similar between groups. On recruitment, 41 patients (84\%) were malnourished but only 25 (61\%) had been referred to a dietitian. Of the participants, 27 (17 control and 10 intervention) were already receiving a high protein/energy diet at the time of recruitment, 39 (18 control and 21 intervention) had baseline data for dietary intake. Only $10(25 \%)$ met estimated energy requirements and $7(18 \%)$ met estimated protein requirements.

In the majority of cases participants had only one PU on enrolment (60\%). Almost half the patients recruited had a stage II PU, and a majority had developed within the previous 7 days. PU were mostly located in the middle torso area (buttock, hip and spine) $(n=28)$. The median PU area of primary PU on recruitment was $1.85 \mathrm{~cm}^{2}$ (range: 0.2-65.3; IQR: 0.84.9) and median PUSH score 8.0 (range: 4.0-17.0; IQR $6.0-11.0)$. However, large PUs $\left(>15 \mathrm{~cm}^{2}\right)$ were only in the standard care group. Overall, there were no significant differences between groups for PU treatment on enrolment, except for the diet prescription. In this case, a majority (68\%) of participants allocated to the standard care group were already receiving a high protein/energy diet as part of their standard care. Pathological data collected also did not indicate any differences between groups.

The median length of stay was 14.0 days (range: 1.0-70.0; IQR: 8.5-25.0) for the study population; 14.0 days (range: 2.0-70.0; IQR: 10.0-31.0) for control group and 14.5 days (range: 1.0-48.0; IQR: 6.2-23.5) for intervention group. Of the 49 participants, 10 patients were discharged by day 7; six patients were less than five days with no data collection beyond baseline. In total, 18 patients were discharged before day $15 ; 35$ by day 22; 41 by day 30; with a further 8 patients staying beyond 30 days. Only 18 patients had their PU heal during admission, with a majority being discharged home before full healing. One patient experienced a worsening of PU during admission. There were only 17 and 14 patients in the control and intervention groups, respectively, able to be included in the final analysis. Despite the high rate of loss to follow-up, there remained no significant differences in baseline characteristics between the two groups.

During the study, participants in the intervention group were reviewed by the dietitian in the majority of cases (74\%) 2-3 times per week, compared with once or never $(83 \%)$ in the standard care group (Table 2 ). This is considered consistent with current practice and the intended intervention. There was no difference in the diet prescription between groups during admission, with most participants being prescribed a high protein/energy diet as part of both standard and intervention protocols. There was no significant difference between groups for patients meeting
Table 2. Characteristics during hospital stay or at discharge for standard care and intervention groups

\begin{tabular}{|c|c|c|}
\hline Parameter & $\begin{array}{r}\text { Standard care } \\
n=25 n(\%)\end{array}$ & $\begin{array}{r}\text { Intervention } \\
n=24 \text { n (\%) }\end{array}$ \\
\hline \multicolumn{3}{|l|}{ Dietitian reviews per week* } \\
\hline None & $8(32)$ & $0(0)$ \\
\hline 1 & $12(48)$ & $3(13)$ \\
\hline $2-3$ & $4(16)$ & $17(71)$ \\
\hline $4-5$ & $0(0)$ & $3(13)$ \\
\hline Data unavailable & $1(4)$ & $1(0.5)$ \\
\hline \multicolumn{3}{|l|}{ Diet @ day 10: } \\
\hline High protein/energy & $18(72)$ & $13(54)$ \\
\hline Standard & $2(8)$ & $0(0)$ \\
\hline Restrictive (for example diabetes) & $3(12)$ & $3(13)$ \\
\hline Data unavailable & $2(8)$ & $8(33)$ \\
\hline \multicolumn{3}{|l|}{ Meet energy intake (30 kcal/kg/day) } \\
\hline \multicolumn{3}{|l|}{ @ day 10: } \\
\hline$<100 \%$ & $7(28)$ & $8(33)$ \\
\hline$>100 \%$ & $5(20)$ & $5(21)$ \\
\hline Data unavailable & $13(52)$ & $11(46)$ \\
\hline \multicolumn{3}{|l|}{ Meet protein intake $(1.5 \mathrm{~g} / \mathrm{kg} /$ day $)$} \\
\hline \multicolumn{3}{|l|}{ @ day 10: } \\
\hline$<100 \%$ & $10(40)$ & $8(33)$ \\
\hline$>100 \%$ & $2(8)$ & $5(21)$ \\
\hline Data unavailable & $13(52)$ & $11(46)$ \\
\hline \multicolumn{3}{|l|}{ Arginine intake @ day 10 *: } \\
\hline $0-4.4 \mathrm{~g} / \mathrm{d}$ & $20(80)$ & $3(13)$ \\
\hline $4.5-8.9 \mathrm{~g} / \mathrm{d}$ & $0(0)$ & $8(33)$ \\
\hline$>9 g / d$ & $1(4)$ & $5(21)$ \\
\hline Data unavailable & $4(16)$ & $8(33)$ \\
\hline \multicolumn{3}{|l|}{ Mobility at discharge: } \\
\hline Cannot reposition & $2(8)$ & $5(21)$ \\
\hline Can reposition but can't walk & $14(56)$ & $7(29)$ \\
\hline Can reposition and can walk & $8(32)$ & $9(38)$ \\
\hline Data unavailable & $1(4)$ & $3(12)$ \\
\hline \multicolumn{3}{|l|}{ PU treatment at discharge: } \\
\hline Moisture donating & $1(4)$ & $2(8)$ \\
\hline Absorb moisture & $12(48)$ & $7(29)$ \\
\hline Antimicrobial & $2(8)$ & $3(13)$ \\
\hline Surgical debridement & $1(4)$ & $2(8)$ \\
\hline Wound open-no dressing & $8(32)$ & $7(29)$ \\
\hline Data unavailable & $1(4)$ & $3(13)$ \\
\hline \multicolumn{3}{|l|}{ Nutritional status (SGA) change*: } \\
\hline No change & $13(52)$ & $6(25)$ \\
\hline Improved & $8(32)$ & $10(42)$ \\
\hline Worsened & $2(8)$ & $0(0)$ \\
\hline Data unavailable & $2(8)$ & $8(33)$ \\
\hline
\end{tabular}


Table 3. Median changes in the primary PU from baseline to day 15

\begin{tabular}{|c|c|c|}
\hline $\begin{array}{l}\text { PU change } \\
\text { measure }\end{array}$ & $\begin{array}{l}\text { Standard Care }(n=17) \\
\text { Median (min, max) IQR }\end{array}$ & $\begin{array}{l}\text { Intervention ( } n=14) \\
\text { Median (min, } \max \text { ) IQR }\end{array}$ \\
\hline PUSH tool score & $\begin{array}{l}-3.0(-13.0,7.0) \\
\text { IQR: }-6.5 \text { to }-1.5\end{array}$ & $\begin{array}{l}4.5(-9.0,4.0) \\
\text { IQR: }-8.0 \text { to }-1\end{array}$ \\
\hline PUSH tool score (\%) & $\begin{array}{l}28.6(-100,100) \\
\text { IQR: }-93.8 \text { to }-14.3\end{array}$ & $\begin{array}{l}-54.2(-100.0,44.4) \\
\text { IQR: }-100.0 \text { to }-16.9\end{array}$ \\
\hline $\mathrm{PU}$ area change $\left(\mathrm{cm}^{2}\right)$ & $\begin{array}{l}-1.7(-18.0,7.1) \\
\text { IQR: }-7.2 \text { to }-0.5\end{array}$ & $\begin{array}{l}-1.4(-5.3,11.3) \\
\text { IQR: }-2.4 \text { to }-0.7\end{array}$ \\
\hline PU area change (\%) & $\begin{array}{l}-74.0(-100.0,592.0) \\
\text { IQR: }-100.0 \text { to }-33.1\end{array}$ & $\begin{array}{l}-86.0(-100.0,332.3) \\
\text { IQR: }-100.0 \text { to }-33.0\end{array}$ \\
\hline
\end{tabular}

estimated energy and protein requirements. Adherence to the wound healing supplement prescription (consumption of recommended volume of supplement per day) was achieved by 14 of 24 patients in the intervention group (58\%). Reasons for nonconcordance included patient dislike of the supplements (seven patients) and/or reported gastrointestinal side effects, including nausea and diarrhoea (three patients).

Fig 2 shows the change in PU area for all participants in the control and intervention groups across admission. The median change in PU area over the study was -1.0 (range: -26.7 to 12.8 ; IQR: -2.25 to $-0.5) \mathrm{cm}^{2}$ for final time point measured for each patient; and -1.4 (range: -180 to 11.3 ; IQR -3.1 to $-0.5) \mathrm{cm}^{2}$ at day 15 . Median change in PUSH score over study was -3.0 (-13.0 to 6.0; IQR -6.0 to -2.0$)$ for the final time point measured for each patient; and -3.0 (range: -13.0 to -7.0 ; IQR -7.0 to -1.0 ) at day 15 . As these numbers were comparable and a majority of patients had available data, day 15 was chosen as a time point representative of expected changes over admission. Changes in PU measures at day 15 for both groups are presented in Table 3 . There was no significant difference between the groups with either measure of PU change. The median reduction in PUSH tool raw score and as a percentage of baseline were somewhat greater in the intervention group, but this was not statistically significant. The median reduction in PU area was greater in the standard care group; however, when converted to a percentage change, the intervention group had a trend to a greater reduction, but this was not statistically significant. All PUSH scores and PU area measurements were strongly correlated $(\mathrm{p}<0.01)$. PUSH change score at day 15 did not correlate with PUSH score on recruitment, however PU area change at day 15 was found to correlate with PU area on recruitment $(\mathrm{p}=0.000)$. Therefore the larger the initial PU area, the greater the change in area measurement.

In bivariate analysis, predictors of greater percentage change in PUSH score and PU area at day 15 included surgical debridement and frequent dietitian reviews; no statistically significant relationship was seen with protein, energy, arginine or supplement intake.

\section{Discussion}

The aim of the study was to investigate the feasibility of recruitment, retention, intervention delivery and outcome measurement in a nutritional intervention intended to promote PU healing in an acute care setting, to inform a future adequately powered RCT. Based on this trial, data are now available to inform effective methodology and sample size for such a study.

Most significantly, the study identified varying demographics and courses of treatment of this patient group who are usually admitted to hospital for reasons other than having a PU, resulting in widely varying length of stay. The median length of stay of patients in the study was 14 days (1-70), with 18 patients discharged before day 15 and 24 discharged before their PU had fully healed. This was an important finding for a feasibility pilot, highlighting the need for systems of follow-up after discharge in future trials to avoid major losses of potentially informative data in the outcome analysis. Change in PU area and score at day 15 were chosen as appropriate outcomes for analysis because complete data were available for a majority of patients and it was reasonable to expect that an intervention effect would be evident within two weeks. While this measure was feasible and provided useful data for analysis, the most meaningful patient outcome would be time to complete healing, emphasising the importance of a design resourced for post-discharge patient follow-up.

Of note, the median reduction in PU area was greater in the standard care group. This was likely due to five large PU randomised to this group, compared with no large PUs in the intervention group. Since PU area change was found to correlate with initial wound size, caution should be taken when analysing PU area changes between groups. To account for the baseline differences in this study, wound area change was converted to a percentage change, and this approach is recommended for future studies. Given PUSH score is an ordinal scale score and there were no differences between groups at recruitment, it was likely not necessary to convert scores to percentage change. Importantly, all PUSH score changes and wound area changes were found to be highly correlated, indicating either measure can be used to measure PU change.

Compared with other researchers ${ }^{14-16}$ the size of PU wounds of patients recruited in this study were generally smaller. There were only several large PU and these were all randomised to the standard care group. This then had implications for the study results and suggests a different process for randomisation is required. It is recommended that in future studies group allocation is stratified by size of PU/ wound.

This study also found limited difference in 
nutritional intake between the control and intervention groups. On recruitment, only $25 \%$ of patients were meeting estimated energy requirements and $18 \%$ estimated protein requirements and this did not change significantly during the study, although numbers were small. Only $58 \%$ of patients prescribed wound healing supplements consumed the recommended quantity of supplement, which is lower than reported in other studies. ${ }^{14,16,17}$

\section{Limitations}

While all attempts were made to blind the research nurse collecting data of which group patients were randomised to, the actual nutritional intervention, including wound healing formulae, were not disguised or hidden and this may be seen as a limitation of the study. In addition, as patients provided informed consent to be included in the study, there may have been positive effect on nutritional intake across both groups, which may have limited between group differences. Future studies need to focus on ensuring effective delivery of the intervention.

Grouping of categories of comorbidities associated with poorer healing or not was less than ideal since mechanisms for effecting wound healing can differ. However, due to the large number of comorbidities and relatively small sample size, it was deemed necessary to keep multiple categories within variables to a minimum. Future studies should ensure categorical groupings are indeed similar.

This study did not show improved healing of PU during hospitalisation with an intensive nutrition intervention, which included prescription of wound healing formulae, compared with standard care; however, this was not the primary aim, nor was the study adequately powered to achieve this outcome. Based on these results, a sample size of 95 patients per group is required to demonstrate a significant difference between groups. This would assume a realistic and clinically meaningful change in PUSH score at day 14 of $30 \%$ (SD 30\%), with $90 \%$ power and type I error probability of 5\% (two tailed), and assume an attrition rate of $40 \%$ to day 14 and allow 20\% extra for measurement error, and another $15 \%$ extra to apply multivariate modelling techniques to adjust for confounding.

There is a need to determine the efficacy of nutrition interventions in the acute hospital environment and future studies need to account for a high rate of attrition due to discharge or transfer and have methodology that allows follow-up of patients after discharge and ideally until complete healing. Larger sample size and longer follow-up would also allow for meaningful survival analysis methodology to be used.

\section{Conclusion}

While the study did not show any significant difference in PU healing with an intensive nutritional intervention that included prescription of wound healing formulae, it does add to the number of studies that have investigated nutritional interventions in PU and/or wound healing that indicate a positive association on wound healing and suggests there may be a role for such a nutrition intervention in wound healing. This pilot study provides data for a future study investigating this topic that is rigorously designed and delivered and adequately powered. Jwc

\section{References}

1 Banks, M., Graves, N.,

Bauer, J., Ash, S.

Malnutrition and pressure ulcer risk in adults in

Australian health care

facilities. Nutrition 2010;

26: 896-901.

2 Shahin, E.S., Meijers,

J.M., Schols, J.M. et al.

The relationship between malnutrition parameters

and pressure ulcers in

hospitals and nursing

homes. Nutrition 2010; 26 :

9, 886-889

3 lizaka, S., Okuwa, M.

Sugama, J., Sanada, H.

The impact of malnutrition

and nutrition-related

factors on the

development and severity

of pressure ulcers in older

patients receiving home

care. Clin Nutr 2010; 29 :

1, 47-53.

4 Stratton, R., Ek, A.,

Moore MEZ, et al. Enteral

nutrtional support in

prevention and treatment of pressure ulcers: A systematic review and meta-analysis. Ageing Res Rev 2005; 4: 3, 422-450.

5 Banks, M., Graves, N.

Bauer, J., Ash, S. Cost

effectiveness of nutrition

support in the prevention

of pressure ulcer. Eur $\mathrm{J}$

Clin Nutr 2013; 67:1, 42-46.

6 Langer, G., Fink, A.

Nutritional interventions

for preventing and treating

pressure ulcers. Cochrane

Database Syst Rev 2014;

6: CD003216

7 Cereda, E., Klersy, C.,

Serioli, M. et al. A

nutritional formula

enriched with arginine,

zinc, and antioxidants for

the healing of pressure

ulcers: a randomized trial.

Ann Intern Med 2015; 162

3, 167-174.

8 Dambach, B., Salle, A.,

Marteau, C. et al. Energy

requirements are not greater in elderly patients suffering from pressure

ulcer. J Am Geriatr Soc 2005; 53: 3, 478-482.

9 Raffoul, W., Far, M.S.,

Cayexu, M.C., Berger,

M.M. Nutritional status

and food intake in nine

patients with chronic

low-limb ulcers and

pressure ulcers:

importance of oral

supplements. Nutrition

2006; 22: 1, 82-88.

10 Wojcik, A., Atkins, M.,

Mager, D.R. Dietary intake

in clients with chronic

wounds. Can J Diet Pract

Res 2011; 72: 2, 77-82

11 Sugama, J., Matsui, Y.,

Sanada, H. et al. A study

of the efficiency and

convenience of an

advanced portable wound

measurement system

(VISITRAK). J of Clin

Nursing 2007; 16: 7 ,

1265-1269.

12 Detsky, A.S.,
McLaughlin, J.R., Baker,

J.P. et al. What is

Subjective Global

Assessment of Nutritional

Status? JPEN 1987; 11: 1

8-13

13 National Pressure

Ulcer Advisory Panel \&

European Pressure Ulcer

Advisory Panel (2014).

Prevention and treatment

of pressure ulcers: Clinica

Practice Guideline.

NPUAP and EPUAP

Avaialble at: http://bit.

y/17A4p4b (accessed 13

June 2016).

14 Cereda, E., Gini, A.,

Pedrolli, C., Vanotti, A.

Disease-specific, versus

standard, nutritional

support for treatment of

pressure ulcers in

institutionalized older

adults: a randomized

Controlled Trial. J Am

Geriatr Soc 2009; 57: 8 ,

1395-1402.

15 Ohura, T., Nakajo, T.,
Okada, S. et al. Evaluation of effects of nutrition intervention on healing of pressure ulcers and nutritional states (randomized controlled trial). Wound Repair Reg 2011; 19: 3, 330-336.

16 van Anholt, R.D.

Sobotka, L., Meijer, E.P. et al. Specific nutritional support accelerates pressure ulcer healing and reduces wound care intensity in nonmalnourished patients. Nutrition 2010;26: 9 , 867-872.

17 Heyman, H., van de Looverbosch, D.E., Meijer, E.P., Schols, J.M. Benefits of an oral nutritional supplement on pressure ulcer healing in long-term care residents. J Wound

Care 2008; 17: 11

476-.480
This article was first published as follows: Banks MD, Ross LJ, Webster J, et al. Pressure ulcer healing with an intensive nutrition intervention in an acute setting: a pilot randomised controlled trial. J Wound Care. 2016; 25(7): 384392. https:// doi. org/10.12968/ jowc. 2016 . 25.7.384 